\title{
Philosophiques
}

\section{Alain Boutot, Heidegger et Platon. Le problème du nihilisme, Paris, Presses Universitaires de France, (Philosophie d'aujourd'hui), 1987, 350 p.}

\section{Georges Leroux}

Volume 19, numéro 1, printemps 1992

URI : https://id.erudit.org/iderudit/027175ar

DOI : https://doi.org/10.7202/027175ar

Aller au sommaire du numéro

Éditeur(s)

Société de philosophie du Québec

ISSN

0316-2923 (imprimé)

1492-1391 (numérique)

Découvrir la revue

Citer cet article

Leroux, G. (1992). Alain Boutot, Heidegger et Platon. Le problème du nihilisme, Paris, Presses Universitaires de France, (Philosophie d'aujourd'hui), 1987, 350 p. Philosophiques, 19(1), 113-122. https://doi.org/10.7202/027175ar d'utilisation que vous pouvez consulter en ligne.

https://apropos.erudit.org/fr/usagers/politique-dutilisation/ 
PHILOSOPHIQUES, VOL. XIX, NUMÉRO I, PRINTEMPS 1992

\title{
ÉTUDES CRITIQUES
}

\begin{abstract}
ALAIN BOUTOT, Heidegger et Platon. Le problème du nihilisme, Paris, Presses Universitaires de France, (Philosophie d'aujourd'hui), 1987. $350 \mathrm{p}$.

par Georges Leroux
\end{abstract}

L'intérêt de Heidegger pour l'histoire de la philosophie n'est pas un caractère accidentel de sa pensée. Il ne l'aborde pas comme l'objet d'un savoir historiographique, mais il la produit comme le cœur même de son entreprise. Pas un instant, il ne doute de la possiblité d'avoir un accès immédiat au texte, et cela hors de la distance et des médiations qui résulteraient de l'éclairage par une discipline auxiliaire. L'érudition des philologues, dont le développement fut très important chez ses contemporains, ne l'intéresse guère. Cet accès direct au texte est, bien au contraire, l'exigence la plus forte de sa pensée dans le milieu même de l'histoire de la philosophie, sans qu'il soit besoin de faire intervenir quelque savoir de cette histoire et de ses objets. Si on compare cette attitude à celle de Hegel, on peut voir mieux néanmoins où Heidegger a voulu placer la limite. Là où Hegel a cherché à produire une réincorporation effective de l'histoire de l'esprit, dont l'effet le plus définitif est l'accomplissement de cette histoire dans une fin de la philosophie, Heidegger accepte le développement d'une histoire différenciée qu'il ne récapitule pas et dont il n'est qu'un relais, peut-être rédempteur. Cette différence insistante est celle qui sépare tout effort dialectique de la tâche de l'interprétation. Pour l'histoire de la philosophie pratiquée par Heidegger, c'est l'interprétation qui est constitutive. Si elle doit être libre de la science historique, dans son accès sans médiation à l'impensé de la métaphysique, elle ne peut être libre de toute contrainte historique.

Cette herméneutique a été abondamment moquée. Sa vulnérabilité lui vient de ce qu'elle expose sans défense le rapport de l'interprétation à des thèses sur l'histoire de l'être, dont l'herméneutique est la méthode même. Il n'est pas indifférent en effet à la pensée de Heidegger que les présocratiques soient disposés comme le recueil de cette 
pensée qui sera oubliée à compter de Platon. La proposition sur l'oubli est doublement constitutive: d'une part, elle s'élabore ellemême comme la forme originaire du nihilisme, mais elle est aussi bien, d'autre part, le motif de l'appel qui rend possible l'exercice de la pensée dans le travail contemporain de l'interprétation. La pensée doit être vigilance et mémoire de la philosophie. Dit autrement, comme le fil de l'interprétation est gardé vivant, puisqu'il est engagé dans la manifestation de l'être, l'herméneutique ne peut être identifiée à une simple méthode de travail historique sur un objet découpé par le temps, délimité dans un temps absous de sa modernité; elle est travail sur le temps de l'interprétation vivante et en cela elle s'expose à partir de la pensée qui la commande. La proposition sur l'oubli n'est donc pas une thèse quelconque d'histoire de la philosophie, c'est la proposition centrale de la doctrine de l'histoire de l'être.

Voici un livre qui se donne pour tâche d'éclairer avec la plus grande rigueur possible le rapport constitutif de Heidegger à Platon. Il s'agit d'un livre important pour deux raisons principales. Insistons d'abord sur le fait que l'auteur n'a pas de travers anti-heideggerien et qu'il n'est pas non plus un épigone lyrique d'une œuvre difficile à commenter. Il a cherché à tenir une position juste et ouverte, dont le postulat est que l'interprétation de Platon par Heidegger est soumise à une rigueur et à une cohérence qui ne sauraient être déstabilisées par le seul exercice de quelque moquerie philologique. $\mathrm{Ce}$ postulat le porte à une attitude d'exposé ouvert qui tranche avec l'hostilité ou le fétichisme qu'on rencontre habituellement, mais il n'empêche pas l'auteur de discuter les critiques les plus importantes, notamment celle de V. Goldschmidt. Il faut ensuite faire remarquer qu'il s'agit du premier ouvrage qui prend en compte la totalité des passages platoniciens disséminés dans l'œuvre publiée de Heidegger, avec le souci d'en restituer la genèse et la progression. Chaque morceau est analysé dans le détail, sa contribution au mouvement d'ensemble est exposée et mise en perspective, et ainsi s'opère un magnifique travail de lecture au terme duquel ce n'est pas tant la métaphysique platonicienne qui se trouve mieux éclairée que son rôle constitutif dans l'histoire de l'être. Platon y apparaît comme une figure focale, qui irradie tout le reste.

Les lacunes et les limites de l'interprétation heideggerienne ne sont pas marquées, dans la mesure où cet effort nous convie précisément à une critique centrée sur le rôle d'une philosophie pour une autre philosophie; l'histoire de la philosophie change en quelque sorte de sens, elle assume une destination herméneutique qui la prive de ses illusions d'objectivité et elle se révèle imprégnée de part en part par la proposition du nihilisme. En cela, l'auteur a raison d'y insister, Heidegger répète paradoxalement le geste de Nietzsche. Il adopte un biais qui est constitutif de sa pensée et qu'il ne sert à rien de désigner comme biais, la question n'étant pas de savoir si Heidegger a raison de représenter Platon comme il le fait, mais 
bien de saisir le rôle de cette représentation dans la doctrine de l'histoire de l'être.

Le thème central par lequel se marque la position de Platon dans l'histoire de l'être est celui du déclin. Une analyse exhaustive du commentaire de la métaphysique platonicienne chez Heidegger doit donc tendre à exposer la signification de ce déclin, à en mesurer les raisons, à jeter toute la lumière possible sur une interprétation de l'histoire de la pensée qui se détermine par l'oubli. Même si les textes consacrés à Platon sont peu nombreux, l'inventaire de l'enseignement de Heidegger révèle une préoccupation quasi constante à travers le questionnement sur le nihilisme. Toujours Platon réintervient obli quement, notamment dans la réflexion sur Nietzsche. De même, très peu de textes cardinaux de Platon semblent avoir sollicité Heidegger: l'allégorie de la Caverne, le passage sur le Bien, quelques passages du Sophiste. Mais cela ne doit pas abuser. Une seule thèse platonicienne engage, dans sa récurrence, l'interprétation du déclin, et elle constitue pour Heidegger l'épitomé du platonisme, le geste par lequel Platon dénature l'effort philosophique originel: la position de l'être en tant qu'étantité (Seiendheit). Se détournant de la vérité de l'être, de l'être en tant qu'être, Platon limite la philosophie à la seule considération de l'essence de l'étant. C'est sur le fond de cette proposition originaire de l'interprétation de Heidegger qu'il faut replacer le sens primitif du nihilisme: le nihilisme, c'est d'abord et avant tout l'oubli de l'être.

C'est donc cette thèse centrale qui est retoumée de toutes les façons dans tous les textes où la pensée de Platon est convoquée. Dans le geste fondateur de la métaphysique, Heidegger cherche à dégager le contenu impensé qui a marqué de son empreinte la suite de la philosophie. L'accès à cette loi cachée ne peut s'effectuer sans violence, l'interprétation doit en effet «forcer" l'oubli et le nihilisme dans la pensée de la présence et de l'idée, elle doit en révéler le pouvoir de négation et d'absence. Il ne faut pas se surprendre, même si on peut le déplorer, de ce que Heidegger revienne toujours aux mêmes motifs du platonisme: ce n'est pas l'ensemble de la pensée de Platon qui le sollicite, mais seulement le détour fondateur du nihilisme. Si donc cette interprétation doit être analysée, ce doit être sur le fond de son propre télos. À ce précepte, le livre de Alain Boutot souscrit entièrement; pour lui, la rigueur et la cohérence de l'approche heideggerienne ne sauraient être mesurés à des critères étrangers au projet de saisie du nihilisme. C'est la portée même de la question de Heidegger qui engage la validité de l'interprétation, même au prix de la violence. Sauf à retirer à une philosophie le droit de l'incorporation de l'autre, ce précepte ne peut être démis. La vérité de l'histoire de la philosophie n'appartient plus aux doctrines philosophiques prises en elles-mêmes, elle appartient à l'élaboration de l'impensé qui s'effectue dans l'interprétation de l'histoire de l'être. Cette élaboration se donne à nous comme une appropriation dont le destin est un dépassement et un salut. 
Le livre se divise en deux parties. La première expose la lecture de Platon chez Heidegger. Dans un premier moment, l'auteur analyse les présupposés de l'idéalisme platonicien, notamment l'identité de l'être et de la présence constante. De cette identité, Platon tire l'exigence de l'idée. Un deuxième moment est consacré à l'idéalisme platonicien proprement dit; on peut le caractériser comme une identification de l'être et de l'a priori, mais aussi comme l'onto-théologie du Bien. Un troisième moment décrit les conséquences de cet idéalisme sur la logique (la question de la vérité), la physique (la question de la chôra) et l'esthétique. La deuxième partie, beaucoup plus courte, analyse la postérité du platonisme: la métaphysique n'est-elle que la répétition historique du platonisme? On cherche à montrer ici comment la condition de possibilité kantienne et la valeur nietzschéenne constituent de telles répétitions. Le livre se clôt par une réflexion sur l'enjeu, pour Heidegger, de la dénonciation de l'histoire du platonisme comme histoire de l'oubli de l'être.

Ce parcours représente certainement la nécessité la plus interne du platonisme: de l'exigence de la présence constante, Heidegger passe à la position de l'essence éternelle de l'étant comme idée. La question: "qu'est-ce que l'étant? " manifeste son caractère déterminant dans toute l'œuvre platonicienne, puisqu'elle conduit inéluctablement à la séparation de l'être et de l'étant et plus profondément à la négation du caractère d'être de l'étant: l'étant sensible est privé du privilège ontologique, puisque la philosophie recherche seulement ce qui est "étamment étant" (c'est ainsi que Heidegger traduit ontôs on). Sur ce point, le livre de Alain Boutot montre une évolution importante dans l'attitude de Heidegger à l'égard de Platon: à l'époque de Etre et Temps, Platon apparaît comme celui qui a posé dans le Sophiste la question du "sens d'être " et Heidegger ne peut que le répéter, tout en montrant les insuffisances de son élaboration quant à la question de la compréhension de l'être par le Dasein. Plus tard cependant, à l'époque de "La doctrine platonicienne de la vérité ", Platon est rejeté: la question sur l'étant est oublieuse de l'être, elle s'abolit dans la détermination métaphysique de l'essence, de l'ousia. C'est cette thèse qui est centrale, citons-la telle que l'expose l'auteur:

L'ousia n'est pas l'être comme tel, mais l'être pensé relativement à l'étant comme étantité commune à tout étant. En pensant l'être comme ousia, Platon inaugure en quelque sorte l'histoire de l'oubli de l'être (p. 53).

Dans cette présentation, dont l'énoncé est récurrent, l'importance du "comme tel » ne doit pas être mésestimée; on laisse entendre en effet que d'une certaine façon, qui ouvre sur le nihilisme mais qui néanmoins demeure une pensée, l'ousia dit quelque chose de l'être, mais non pas comme tel. La préséance du " comme tel " sur toute autre forme d'appréhension est déjà le signe du privilège des présocratiques qui, ignorant la différence ontologique, ont accès à une appréhension non sédimentée. 
De cette première détermination on passe à la position de l'horizon du temps: l'être signifie être présent constamment, et l'ousia devient donc parousia. L'idée est éternelle et Heidegger insiste sur le rapport problématique de cette éternité au temps de la compréhension; cette critique l'amène à juger sévèrement la conception platonicienne de la temporalité de l'être, dans la mesure où elle demeure prisonnière d'une représentation cosmologique et mathématique du temps du " maintenant ", injustement oublieuse d'une temporalité originaire à laquelle faisait signe le concept du kaîros.

Il appartient au concept de l'idée (eidos, idea) de consommer ce qui n'était jusque là que présupposés. Alain Boutot montre nettement les différences entre l'idée platonicienne et l'idée moderne: il ne s'agit pas de représentations, ayant leur lieu dans un sujet. Pour Platon, et les Grecs en général, Heidegger le répète souvent, " l'homme n'est absolument pas sujet ". L'idée est identique à l'eidos: elle exprime l'aspect des choses sous le rapport de leur quiddité. Heidegger se montre donc attentif à ce qui se joue dans la langue de la métaphore de l'aspect visible: "On n'en a pas fini avec l'extraordinaire..." écrit-il au sujet de ce paradoxe de l'aspect invisible de la chose. Bien étrangère à la représentation, l'idée platonicienne trouve ici son interprétation comme " auto-présentation de l'étant lui-même". Boutot insiste à juste titre sur la coupure qu'instaure cette interprétation avec le néo-kantisme dominant à son époque. En-deça de la condition de possibilité de la pensée, Heidegger veut redécouvrir chez Platon la manifestation.

Comment la détermination de l'idée est-elle la cause du déclin? La réponse se tient dans l'écart qui la sépare de la physis, "mot fondamental " (Grundwort) de la pensée grecque des origines. Boutot consacre une belle section de son ouvrage à exposer l'interprétation heideggerienne de ce terme originaire, dans lequel se condensent toutes les significations primitives de l'être: l'éclosion à partir de soi même, le paraître s'épanouissant. Dans quelle mesure l'idée qui procède de la physis en vient-elle à produire la séparation constitutive de la métaphysique? Heidegger n'écrit-il pas, à plusieurs reprises, que Platon ne rompt point avec le premier commencement de la pensée? Le déclin s'annonce quand l'idée prend la place de l'essence originaire, quand elle se substitue à la physis au lieu d'en demeurer le déploiement: "Ce qui reste décisif, écrit Heidegger, ce n'est pas que la physis ait été caractérisée comme idea, c'est que l'idea s'installe comme l'interprétation unique et déterminante de l'être". Ou encore: "Le visage que fait la chose est maintenant décisif, non plus la chose elle-même " [ Einführung in die Metaphysik, Gesamtausgabe, Bd 40, p. 191 et p. 192; trad. fr., pp. 186-87]. Comme toute la signification de ce déplacement n'apparaît que dans le rapport de l'idée à ce qu'elle vient occulter, l'interprétation de Heidegger demeure suspendue à la doctrine de l'être. On verra que le livre de Boutot ne cherche pas à élucider ce rapport, dont 
l'indétermination énigmatique appellerait un deuxième temps de sa critique.

Il est important de bien préciser à ce stade la signification du nihilisme: la chose qui s'épanouissait dans la présence se trouve maintenant niée, elle n'est plus rien et elle est reléguée derrière son aspect eidétique. Cette première version du nihilisme peut donc se présenter ainsi: "Tout idéalisme à vrai dire, dans la mesure où il accorde la préséance à l'idéel et à l'idéal sur le réel, sur la res, est d'une certaine façon "nihiliste". Platon, le premier idéaliste, est aussi et corrélativement le premier nihiliste" (p. 132). Cette interprétation, en apparence paradoxale, n'est pas remise en cause par Boutot, qui s'applique plutôt à en montrer la fécondité pour une histoire de l'être. Elle se confirme par l'analyse de l'a priori: l'être apparaît depuis Platon comme ce qui est antérieur (anté-cédent, Vorherige) à l'étant, il est le premier (proteron). En abandonnant l'être à ce statut apriorique, la métaphysique renonce à le penser comme tel; elle s'accomode de sa séparation et ainsi se trouve renforcé le nihilisme de l'idée.

Cette interprétation ne serait pas achevée si elle se révélait incapable d'intégrer dans le nihilisme ce qui semble d'emblée en constituer la réfutation: l'idée du Bien. Comment concilier en effet le nihilisme avec l'affirmation positive du livre VI de la République? Quelle est, dit autrement, la négativité du Bien quand on cherche à en situer l'effet dans l'histoire de l'être? Quel être est nié par le Bien? Heidegger en situe l'approche au niveau de ce qui est "apte " (das Taugliche): le bien, c'est posséder une aptitude déterminée. On se trouve dès lors au-delà de toutes les connotations morales et théologiques de l'interprétation chrétienne de ce passage célèbre. Est-ce suffisant pour comprendre l'essence "agathoïde " de l'idea platonicienne? En quel sens, notamment, ne satisfait-elle pas aux exigences d'au-delà dans lesquelles Heidegger invite à reconnaître la pensée de l'être? Boutot montre qu'en fait Heidegger refuse à la transcendance du Bien le dépassement du plan ontique: elle demeure en effet une idée, elle ne permet aucune ouverture sur un au-dela de l'étantité. Il reviendra à Plotin de proposer une interprétation du Bien qui puisse être compatible avec la requête de Heidegger. Mais celui-ci, peut-être en raison des rapports étroits entre le néoplatonisme et le christianisme, n'a jamais considéré l'Un plotinien comme un dépassement des apories de l'ousia.

Sur ce point central de son interprétation, Heidegger semble avoir beaucoup hésité et un des mérites de cette section du livre est de le montrer avec netteté. On peut distinguer avec l'auteur trois variantes: en 1929, dans Vom Wesen des Grundes, Heidegger voit la transcendance du Bien comme une préfiguration de la transcendance du Dasein; en 1935, dans l'Introduction à la métaphysique, Heidegger insiste sur la transcendance du devoir sur l'être et enfin, en 1940, dans Le nihilisme européen, la transcendance de l'essence sur ce dont elle est l'essence. Ici réside l'enjeu de l'ensemble de l'interpré- 
tation, puisque pour Heidegger il est essentiel d'accentuer le caractère d'idée de l'idée du Bien, au détriment de son caractère exceptionnel de cause transcendante. Plusieurs textes témoignent de ce travail intensif sur le Bien comme ce qui rend apte l'étant à être étant: "Être se montre dans le caractère du rendre possible et du conditionner. Ici est effectué le pas décisif pour toute métaphysique par quoi le caractère "a priori" de l'être reçoit du même coup la vertu d'être condition " [Nietzsche, II, p. 226; trad. fr., p. 180]. Cette lecture laisse de côté non seulement les métaphores de la causalité (paternité, fécondité), mais encore les expressions mimétiques (trace, empreinte, participation) qui engagent si fortement la métaphysique platonicienne vers les solutions monistes qui interviendront après. On aurait tort cependant de croire que la critique de Heidegger s'exerce à l'endroit du dualisme qui caractérise les thèses de la causalité; dans ce dualisme, ce n'est pas tant l'écart ou la séparation qui fait obstacle à la pensée de l'être, puisque celle-ci est toujours déjà intriquée dans le jeu de la différence, mais l'oubli de cette différence dans le privilège exorbitant de l'ousia.

Même si l'auteur garde ici la réserve qu'il s'est donnée pour consigne de lecture, on ne peut s'empêcher de noter à quelles limites extrêmes l'interprétation du Bien comme "condition " tend le concept du nihilisme; dans la proposition de l'onto-théologie, en effet, il s'en faut de peu que l'ensemble du modèle n'éclate. Ce n'est plus seulement l'étantité de l'essence qui "annule " ou " néantise » l'être, en ruinant l'indissociable unité originelle de la manifestation de la physis; ce n'est plus le caractère a priorique et idéel de l'idée, mais c'est le surcroît même que Platon a voulu conférer au monde des Formes intelligibles qui se trouve réinterprété dans la conceptualité kantienne de la condition de possibilité. Le néo-kantisme qui avait été rejeté sur le plan de l'idée reçue comme aspect et non comme représentation fait ici retour de manière non critique.

Cela est-il acceptable? Il faut peut-être sur ce point briser avec la neutralité de l'auteur: ce qui peut être entendu de l'idée ne saurait l'être en général du Bien, au risque de parvenir à une torsion inadmissible. En effet, si le Bien est pure condition de possibilité, on devra accepter qu'il finisse par s'effacer derrière l'étant, satisfaisant ainsi au thème de l'oubli. L'être comme bien devient donc selon Heidegger pur supplément, "ce qui au regard de la prépondérance de l'étant est simplement toléré en tant que condition de possibilité de l'étant " [Nietzsche, II, p. 229; trad. fr., p. 182]. Cette conclusion est incompatible avec tout ce qui est central dans la République.

Deux choses frappent à ce stade de notre lecture: d'abord, il est remarquable que des prémisses de lecture assez rigoureuses se montrent fécondes pour faire valoir la lecture que fait Heidegger de la thèse platonicienne des Formes intelligibles (c'est ainsi, selon les suggestions répétées de Cherniss et de Brisson qu'il faudrait 
toujours qualifier l'eidos) et que ces mêmes prémisses produisent des absurdités lorsqu'on les applique à la thèse métaphysique du Bien. Cette question, mal débrouillée dans l'ouvrage, semble tributaire de la rémanence des concepts néo-kantiens dans les textes qui gravitent autour du commentaire de Nietzsche. Ce n'est pas n'importe quelle différence entre l'être et l'étant qui peut être rendue responsable de l'avènement du nihilisme; et plus profondément, peut-on accepter de penser comme nihilisme, même dans le sens que donne Heidegger à ce terme, la thèse même par laquelle Platon a cherché à dépasser le simple dualisme ontologique des idées en lui donnant un fondement? Là où il y aurait une ouverture pour montrer les déplacements opérés par Heidegger et évaluer leurs conséquences, Boutot se limite à un exposé dont l'abstention est sans doute conforme au principe de sa lecture, mais sur ce point central, il faut le déplorer.

La présentation de la constitution de l'onto-théologie laisse en effet loin derrière la question du nihilisme, quelle que soit la version qu'on en adopte. Il y a ici, pour ainsi dire, un abîme sur lequel l'interprétation de Platon s'est développée et qui ne saurait être simplement assujetti au nihilisme ontologique. Même si on accepte de laisser de côté tous les aspects constitutifs de la pensée de Platon qui sont nécessaires à une interprétation cohérente, comme c'est le cas par exemple de l'âme, véritable acteur du drame platonicien, mais absolument absent ici, on ne peut, pour suivre Heidegger, replier toute la différence ontologique induite par une doctrine des formes sur une interprétation qui doit se soutenir du néo-kantisme pour en maintenir le nihilisme. L'âme n'est que le plus central des éléments mis dans la marge, il faudrait aussi prendre en compte les aspects narratifs de la République dont l'interprétation de la causalité dépend plus qu'on ne le croit d'ordinaire. Ces éléments prennent encore plus de relief quand on les associe au Phèdre et au Timée.

Dans la deuxième partie de l'ouvrage, l'auteur étend à la considération de l'ensemble de l'histoire de la métaphysique les analyses qui ont précédé. C'est l'ensemble de la métaphysique qui vient à servir la catégorie du nihilisme: toute la philosophie est ancrée dans l'histoire de l'oubli de l'être, à proportion précisément qu'elle n'a fait que poursuivre le geste de Platon. Essentiellement, Heidegger renvoie ici à Kant et Nietzsche. La défaillance ou la défection de l'être se perpétue dans la différence des conditions de possibilité de l'expérience et de l'objet de l'expérience, dans la différence de la valeur et de la vie. "Dans chacun de ces couples, qui représentent autant d'étapes décisives dans l'enfouissement de la première pensée grecque, la vérité de l'être comme tel reste absente " (p. 261). La question soulevée par ce "comme tel " est insistante, mais elle ne trouverait sa résolution que dans un développement sur l'être absent. Même la proposition néoplatonicienne d'une hénologie ne saurait être rapprochée de la visée de Heidegger et l'auteur, à juste titre, fait ici état des réserves de Pierre Hadot. 
C'est la figure de Nietzsche qui reçoit l'éclairage le plus important, dans la mesure où elle semble avoir inspiré la lecture de Heidegger. Les différences d'approche se montrent finalement plus importantes que les similitudes. Au-delà de leur commune détermination du platonisme comme nihilisme et au-delà de leur admiration des présocratiques, Heidegger ne voit chez Nietzsche qu'épuisement et achèvement du nihilisme. L'oubli de l'oubli de l'être se consomme dans l'illusoire renversement des valeurs, dans l'illusoire renversement du platonisme. Cette inversion demeure prisonnière de la différence et en ce sens le nihilisme de Nietzsche ne peut être identifié au nihilisme que retrace Heidegger depuis Platon, l'oubli de l'être. Plus profondément, Nietzsche n'a pas vu la signification du nihilisme platonicien en tant que "demeurer manquant de l'être ». En conséquence, il s'est rabattu sur une négation banale de l'étant. En remontant aux fondements ontologiques de l'interprétation nietzschéenne de la métaphysique, Heidegger en demeure-t-il tributaire? La réponse de Boudot demeure ici très approximative: il hésite à trancher entre la différence instituée par l'interprétation du nihilisme et les ressemblances dans le rapport à la pensée grecque. Cette hésitation constitue de fait la matière d'une autre recherche.

Si l'attitude de Nietzsche peut être dépassée, ce ne peut être que par le travail de l'interprétation comme remémoration; contre l'oubli institué par le platonisme, oubli qui se redouble dans le fait qu'il est lui-même occulté par le seul souci de l'étant, l'interprétation doit chercher à produire des effets de reconnaissance. Heidegger en parle comme d'une tâche: délaisser le platonisme, se remettre à l'écoute de ce qui a été oublié. Dans la visée de cette tâche se trouve réintroduit le dévoilement, c'est-à-dire la positivité de ce qui a été nié par la doctrine platonicienne de la vérité. Le platonisme n'apparaît finalement comme époque de l'histoire de l'être que pour être renversé et dépassé. L'importance de son interprétation vient donc de ce qu'il constitue la pure négativité de la pensée de l'être. Ce que le délaissement de cette négativité permet de penser, seule une interprétation de l'être heideggerien permettrait de le formuler. Le livre d'Alain Boudot s'achève sur cette perspective, il lui suspend toute sa lecture du platonisme, mais il demeure sur le seuil d'une élaboration qui, délaissant Platon, ferait son chemin dans l'œuvre de Heidegger.

Malgré des réserves qui ont trait fondamentalement aux principes de l'herméneutique heideggerienne, ce livre accomplit un travail qui s'imposait depuis longtemps: mettre à nu l'extraordinaire réappropriation de la métaphysique platonicienne accomplie par Heidegger, au titre même de la constitution de l'interprétation. La violence faite aux textes s'efface dès lors qu'on admet les principes de cette réappropriation: l'herméneutique se constitue de cette violence, elle instaure un dialogue avec le texte ancien dont la légitimité ne provient pas des protagonistes. Ni l'interprète, ni l'interprété ne se donnent 
comme dépositaires d'une vérité qu'il s'agirait d'exhumer. Bien plutôt, le mouvement d'avancée et de retrait, jusque dans la dissimulation, que Heidegger prédique de l'être semble commander le jeu même de la philosophie; c'est à lui, en ce sens, qu'appartiennent l'appel et la convocation dont l'interprète cherche à restituer l'écoute. La doctrine de l'être circonvient donc entièrement le projet interprétatif et interdit de le mesurer autrement. Le livre d'Alain Boudot se montre parfaitement fidèle à cette situation de l'interprétation. Il fait voir l'inutilité d'entreprises de critique se réclamant de critères étrangers à cette herméneutique, en même temps qu'il éclaire la rigueur et la cohérence de cette herméneutique prise en elle-même. En quittant ce livre, la polarité de l'interprétation de l'œuvre platonicienne se fige, en se condensant dans la double figure de Plotin et de Heidegger: dans l'exercice d'une même violence, Plotin a produit l'interprétation qui allait soutenir philosophiquement le christianisme et Heidegger a voulu achever la déconstruction qui ouvrirait la possibilité d'une autre pensée, encore à venir.

Département de philosophie

Université du Québec à Montréal 